\title{
Canonical seesaw implication for two-component dark matter
}

\author{
Phung Van Dong $\odot,{ }^{*}$ Cao H. Nam, ${ }^{\dagger}$ and Duong Van Loi $\odot^{\ddagger}$ \\ Phenikaa Institute for Advanced Study and Faculty of Basic Science, Phenikaa University, \\ Yen Nghia, Ha Dong, Hanoi 100000, Vietnam
}

(Received 18 July 2020; accepted 29 April 2021; published 18 May 2021)

\begin{abstract}
We show that the canonical seesaw mechanism implemented by the $U(1)_{B-L}$ gauge symmetry provides two-component dark matter naturally. The seesaw scale that breaks $B-L$ defines a residual gauge symmetry to be $Z_{6}=Z_{2} \otimes Z_{3}$, where $Z_{2}$ leads to the usual matter parity, while $Z_{3}$ is newly recognized, transforming quark fields nontrivially. The dark matter components-that transform nontrivially under the matter parity and $Z_{3}$, respectively — can gain arbitrary masses, despite the fact that the $Z_{3}$ dark matter may be heavier than the light quarks $u, d$. This dark matter setup can address the XENON1T anomaly recently observed and other observables, given that the dark matter masses are nearly degenerate, heavier than the electron and the $B-L$ gauge boson $Z^{\prime}$, as well as the fast-moving $Z_{3}$ dark matter has a large $B-L$ charge, while the $Z^{\prime}$ is viably below the beam dump experiment sensitive regime.
\end{abstract}

DOI: 10.1103/PhysRevD.103.095016

\section{MOTIVATION}

Neutrino mass and dark matter are the two big questions in science, which require the new physics beyond the standard model [1].

It is well established that the canonical seesaw mechanism can generate appropriate small neutrino masses through the exchange of heavy Majorana right-handed neutrino singlets, $\nu_{a R}$ for $a=1,2,3$, added to the standard model [2-10]. However, the canonical seesaw in its simple form does not naturally address the dark matter issue, unless some dark matter stability condition or parameter finetuning is ad hoc imposed.

The simplest gauge completion of the seesaw mechanism with $U(1)_{B-L}$ can provide a natural origin for the existence of the right-handed neutrinos and the righthanded neutrino mass scale [11-13]. This work shows that such theory manifestly yields a novel consequence of two-component dark matter, properly solving the recent XENON1T excess [14].

\footnotetext{
${ }^{*}$ Corresponding author. dong.phungvan@phenikaa-uni.edu.vn nam.caohoang@phenikaa-uni.edu.vn

"loi.duongvan@phenikaa-uni.edu.vn
}

Published by the American Physical Society under the terms of the Creative Commons Attribution 4.0 International license. Further distribution of this work must maintain attribution to the author(s) and the published article's title, journal citation, and DOI. Funded by SCOAP ${ }^{3}$.

\section{DESCRIPTION OF THE MODEL}

Indeed, the full gauge symmetry takes the form,

$$
S U(3)_{C} \otimes S U(2)_{L} \otimes U(1)_{Y} \otimes U(1)_{B-L} .
$$

Here the right-handed neutrino fields $\nu_{a R}$ transforming under the gauge symmetry as

$$
\nu_{a R} \sim(1,1,0,-1)
$$

are required in order to cancel the $[\text { Gravity }]^{2} U(1)_{B-L}$ and $\left[U(1)_{B-L}\right]^{3}$ anomalies. Additionally, a scalar singlet transforming under the gauge symmetry as

$$
\chi \sim(1,1,0,2)
$$

must be presented to break $U(1)_{B-L}$ for the model consistency, simultaneously generating the right-handed neutrino masses or the seesaw scale.

As usual, let us assign the standard model lepton, quark, and Higgs representations with respect to the new gauge symmetry to be,

$$
\begin{gathered}
l_{a L}=\left(\begin{array}{c}
\nu_{a L} \\
e_{a L}
\end{array}\right) \sim\left(1,2,-\frac{1}{2},-1\right), \\
e_{a R} \sim(1,1,-1,-1), \\
q_{a L}=\left(\begin{array}{c}
u_{a L} \\
d_{a L}
\end{array}\right) \sim\left(3,2, \frac{1}{6}, \frac{1}{3}\right),
\end{gathered}
$$




$$
\begin{gathered}
u_{a R} \sim\left(3,1, \frac{2}{3}, \frac{1}{3}\right), \\
d_{a R} \sim\left(3,1,-\frac{1}{3}, \frac{1}{3}\right), \\
\phi=\left(\begin{array}{c}
\phi^{+} \\
\phi^{0}
\end{array}\right) \sim\left(1,2, \frac{1}{2}, 0\right) .
\end{gathered}
$$

The scalar multiplets develop vacuum expectation values (VEVs), such as

$$
\langle\chi\rangle=\frac{\Lambda}{\sqrt{2}}, \quad\langle\phi\rangle=\left(\begin{array}{c}
0 \\
\frac{v}{\sqrt{2}}
\end{array}\right),
$$

satisfying

$$
\Lambda \gg v=246 \mathrm{GeV} .
$$

The Yukawa Lagrangian includes

$$
\begin{aligned}
\mathcal{L} & \supset h_{a b}^{\nu} \bar{l}_{a L} \tilde{\phi} \nu_{b R}+\frac{1}{2} f_{a b}^{\nu} \bar{\nu}_{a R}^{c} \chi \nu_{b R}+\text { H.c. } \\
& \supset-\frac{1}{2}\left(\bar{\nu}_{a L} \bar{\nu}_{a R}^{c}\right)\left(\begin{array}{cc}
0 & m_{a b} \\
m_{b a} & M_{a b}
\end{array}\right)\left(\begin{array}{c}
\nu_{b L}^{c} \\
\nu_{b R}
\end{array}\right)+\text { H.c. },
\end{aligned}
$$

where

$$
m_{a b}=-h_{a b}^{\nu} \frac{v}{\sqrt{2}}, \quad M_{a b}=-f_{a b}^{\nu} \frac{\Lambda}{\sqrt{2}}
$$

Hence, the canonical seesaw is naturally recognized in the $U(1)_{B-L}$ gauge completion given that $v \ll \Lambda$ or $m \ll M$, yielding the observed neutrino $\left(\sim \nu_{a L}\right)$ masses to be

$$
m_{\nu}=-m M^{-1} m^{T}=h^{\nu}\left(f^{\nu}\right)^{-1}\left(h^{\nu}\right)^{T} \frac{v^{2}}{\sqrt{2} \Lambda},
$$

while the heavy neutrinos $\left(\sim \nu_{a R}\right)$ obtain large masses at the $B-L$ breaking scale, $M \sim \Lambda$.

The neutrino oscillation data implies $m_{\nu} \sim 0.1 \mathrm{eV}$ [1], which leads to

$$
\Lambda \sim\left[\left(h^{\nu}\right)^{2} / f^{\nu}\right] 10^{14} \mathrm{GeV}
$$

The seesaw scale $\Lambda$ is close to the grand unification scale if $\left(h^{\nu}\right)^{2} / f^{\nu} \sim 1$. If $\left(h^{\nu}\right)^{2} / f^{\nu}$ is sufficiently small, say $f^{\nu} \sim 1$ and $h^{\nu} \sim 10^{-5.5}-10^{-5}$ proportional to the electron Yukawa coupling, we derive $\Lambda \sim 1-10 \mathrm{TeV}$, in agreement to the collider bounds [1].

All the above results have been established in the literature. However, a proper realization of residual gauge symmetry of $B-L$ and its implication for dark matter have not emerged yet. Let us call the reader's attention to previous works [15-24] relevant to this proposal.

\section{RESIDUAL SYMMETRY AND DARK MATTER}

The symmetry breaking scheme is obtained as

$$
\begin{gathered}
S U(3)_{C} \otimes S U(2)_{L} \otimes U(1)_{Y} \otimes U(1)_{B-L} \\
\downarrow \Lambda \\
S U(3)_{C} \otimes S U(2)_{L} \otimes U(1)_{Y} \otimes R \\
\downarrow v \\
S U(3)_{C} \otimes U(1)_{Q} \otimes R
\end{gathered}
$$

Here the electric charge is related to the isospin and hypercharge as $Q=T_{3}+Y . R$ is a residual symmetry of $U(1)_{B-L}$ that conserves the $\chi$ vacuum, although this vacuum $\langle\chi\rangle=\Lambda / \sqrt{2} \neq 0$ breaks $B-L$ by two units. As being a $U(1)_{B-L}$ transformation, $R=e^{i \alpha(B-L)}$ where $\alpha$ is a transforming parameter. The vacuum conservation condition $R\langle\chi\rangle=\langle\chi\rangle$ leads to $e^{i \alpha(2)}=1$, or equivalently $\alpha=k \pi$ for $k$ integer. Hence, the residual symmetry is

$$
R=e^{i k \pi(B-L)}=\left(e^{i \pi(B-L)}\right)^{k}
$$

It is noted that the transformation with $k$ is conjugated to that with $-k$, i.e., $R^{\dagger}=\left(e^{i \pi(B-L)}\right)^{-k}=R^{-1}$.

Let us commonly denote $A$ to be the gauge fields associated with the gauge symmetry in (1). The $R$ values of all fields are collected in Table I. From this table, we derive that $R=1$ for the minimal value of $|k|=6$ and for every field, except the identity $k=0$. Hence, the residual symmetry $R$ is automorphic to

$$
Z_{6}=\left\{1, p, p^{2}, p^{3}, p^{4}, p^{5}\right\}
$$

where $p \equiv e^{i \pi(B-L)}$ and $p^{6}=1$. Further, we factorize

$$
Z_{6}=Z_{2} \otimes Z_{3}
$$

where

$$
Z_{2}=\left\{1, p^{3}\right\}
$$

is the invariant (or normal) subgroup of $Z_{6}$, while

$$
Z_{3}=Z_{6} / Z_{2}=\left\{Z_{2},\left\{p, p^{4}\right\},\left\{p^{2}, p^{5}\right\}\right\}
$$

TABLE I. $R$ values of leptons, quarks, and bosons, where the generation and left/right chirality indices are omitted since the relevant fields have the same $R$ value.

\begin{tabular}{lccc}
\hline \hline Field & $(\nu, e)$ & $(u, d)$ & $(\phi, \chi, A)$ \\
\hline$R$ & $(-1)^{-k}$ & $e^{i k \pi / 3}$ & 1 \\
\hline \hline
\end{tabular}


TABLE II. Field representations under the residual symmetry $R=Z_{2} \otimes Z_{3}$.

\begin{tabular}{lccc}
\hline \hline Field & $(\nu, e)$ & $(u, d)$ & $(\phi, \chi, A)$ \\
\hline 1 & 1 & 1 & 1 \\
$p^{3}$ & -1 & -1 & 1 \\
$Z_{2}$ & $\underline{1}_{2}^{\prime}$ & $\underline{1}_{2}^{\prime}$ & $\underline{1}_{2}$ \\
$p$ & -1 & $-w^{2}$ & 1 \\
$p^{4}$ & 1 & $w^{2}$ & 1 \\
$p^{2}$ & 1 & $w$ & 1 \\
$p^{5}$ & -1 & $-w$ & 1 \\
$Z_{3}$ & $\underline{1}_{3}$ & $\underline{1}_{3}^{\prime}$ & $\underline{1}_{3}$ \\
\hline \hline
\end{tabular}

is the quotient group of $Z_{6}$ by $Z_{2}$. Thus, the theory automatically conserves both residual symmetries $Z_{2}$ and $Z_{3}$ after symmetry breaking.

We affix the subscripts 2,3 to a $N$-dimensional representation $\underline{N}$ if it is viable, say $\underline{N}_{2,3}$, in order to indicate to those of $Z_{2,3}$, respectively. The field representations under $Z_{2}$ and $Z_{3}$ are computed in Table II, where $w \equiv e^{i 2 \pi / 3}$ is the cube root of unity. Here note that $Z_{2}$ has two (1-dimensional) irreducible representations, $\underline{1}_{2}$ according to $p^{3}=1$ and $\underline{1}_{2}^{\prime}$ according to $p^{3}=-1$, whereas $Z_{3}$ has three (1-dimensional) irreducible representations, $\underline{1}_{3}$ according to $\left(p^{2}, p^{5}\right)=(1,1)$ or $(1,-1)$, $\underline{1}_{3}^{\prime}$ according to $\left(p^{2}, p^{5}\right)=(w, w)$ or $(w,-w)$, and $\underline{1}_{3}^{\prime \prime}$ according to $\left(p^{2}, p^{5}\right)=\left(w^{2}, w^{2}\right)$ or $\left(w^{2},-w^{2}\right)$, which are independent of $p^{3}$ values, 1 or -1 , that identify $Z_{6}$ elements in a coset of the quotient group, respectively. ${ }^{1}$ The representation $\underline{1}_{3}^{\prime \prime}$ is not presented for the existing fields, but the antiquarks $\left(u^{c}, d^{c}\right)$ belong to $\underline{1}_{3}^{\prime \prime}$ under $Z_{3}$.

For brevity, the quotient group can be defined as

$$
Z_{3}=\left\{[1],\left[p^{2}\right],\left[p^{4}\right]\right\},
$$

where each (coset) element $[x]$ consists of two elements of $Z_{6}$, the characteristic $x$ and the other $p^{3} x$, as multiplied by $p^{3}$. Hence, $[1]=\left[p^{3}\right]=Z_{2},[p]=\left[p^{4}\right]=\left\{p, p^{4}\right\}$, and $\left[p^{2}\right]=\left[p^{5}\right]=\left\{p^{2}, p^{5}\right\}$. Because of $\left[p^{4}\right]=\left[p^{2}\right]^{2}=\left[p^{2}\right]^{*}$, the $Z_{3}$ group is completely generated by

$$
\left[p^{2}\right]=\left[e^{i 2 \pi(B-L)}\right]=\left[w^{3(B-L)}\right] .
$$

That said, the $Z_{3}$ irreducible representations $\underline{1}_{3}, \underline{1}_{3}^{\prime}$, and $\underline{1}_{3}^{\prime \prime}$ are simply determined by $\left[p^{2}\right]=[1] \rightarrow 1,\left[p^{2}\right]=[w] \rightarrow w$, and $\left[p^{2}\right]=\left[w^{2}\right] \rightarrow w^{2}$, respectively. Here, the intermediate $Z_{6}$ representations [ $r$ ] consists of $r$ and $\pm r$ as multiplied by $p^{3}= \pm 1$ respectively, which are homomorphic to that of $Z_{3},[r]=\{r, \pm r\} \rightarrow r$.

\footnotetext{
${ }^{1}$ The nontrivial representations of $Z_{3}$ obey $\underline{1}_{3}^{\prime} \otimes \underline{1}_{3}^{\prime \prime}=$ $\underline{1}_{3},\left(\underline{1}_{3}^{\prime}\right)^{3}=\left(\underline{1}_{3}^{\prime \prime}\right)^{3}=\underline{1}_{3},\left(\underline{1}_{3}^{\prime}\right)^{*}=\underline{1}_{3}^{\prime \prime}=\left(\underline{1}_{3}^{\prime}\right)^{2}$, and $\left(\underline{1}_{3}^{\prime \prime}\right)^{*}=\underline{1}_{3}^{\prime}=$ $\left(\underline{1}_{3}^{\prime \prime}\right)^{2}$, whereas that of $Z_{2}$ satisfies $\left(\underline{1}_{2}^{\prime}\right)^{2}=\underline{1}_{2}$ and $\left(\underline{1}_{2}^{\prime}\right)^{*}=\underline{1}_{2}^{\prime}$.
}

TABLE III. Field representations under the alternative residual symmetry $P \otimes Z_{3}$, where note that the matter parity group $P$ is isomorphic to a $Z_{2}$ group generated by $P_{M}$, while the quotient group $Z_{3}$ is generated by $\left[p^{2}\right]$.

\begin{tabular}{lccc}
\hline \hline Field & $(\nu, e)$ & $(u, d)$ & $(\phi, \chi, A)$ \\
\hline$P_{M}$ & 1 & 1 & 1 \\
$P$ & $\underline{1}_{P}$ & $\underline{1}_{P}$ & $\underline{1}_{P}$ \\
{$\left[p^{2}\right]$} & 1 & $w$ & 1 \\
$Z_{3}$ & $\underline{1}_{3}$ & $\underline{1}_{3}^{\prime}$ & $\underline{1}_{3}$ \\
\hline \hline
\end{tabular}

Since the spin parity $P_{S}=(-1)^{2 s}$ is always conserved by the Lorentz symmetry, we can conveniently multiply the residual symmetry $R=Z_{2} \otimes Z_{3}$ with spin-parity group $S=\left\{1, P_{S}\right\}$ to perform

$$
R \rightarrow R \otimes S=\left(Z_{2} \otimes S\right) \otimes Z_{3},
$$

where $Z_{3}$ is retained as the quotient group. The new invariant subgroup $Z_{2} \otimes S$ defines a matter parity

$$
P_{M}=p^{3} \times P_{S}=(-1)^{3(B-L)+2 s},
$$

analogous to the $R$-parity in supersymmetry.

Because of $P_{M}^{2}=1$, we have $P=\left\{1, P_{M}\right\}$ to be a group of matter-parity symmetry by itself, which is an invariant subgroup of $Z_{2} \otimes S$. Therefore, we factorize

$$
R \otimes S=\left[\left(Z_{2} \otimes S\right) / P\right] \otimes P \otimes Z_{3} .
$$

Here $\left(Z_{2} \otimes S\right) / P=\left\{P,\left\{p^{3}, P_{S}\right\}\right\}$ is conserved, if $P_{M}$ is conserved. Therefore, instead of $R \otimes S$, we can consider an alternative residual symmetry which is contained in

$$
R \otimes S \supset P \otimes Z_{3},
$$

where the quotient group $\left(Z_{2} \otimes S\right) / P$ is neglected, since the theory automatically preserves it. Of course, the theory conserves both $P$ and $Z_{3}$, under which the representations under these groups are given in Table III, in which the subscript ${ }_{P}$ indicates to the representations of the matter parity group $(P)$.

Hence, the model provides a natural stability mechanism for two-component dark matter, in which a dark matter component transforms nontrivially under the matter parity group $P \cong Z_{2}$ [which should not be confused with the first $Z_{2}$ in (19)], i.e., in $\underline{1}_{P}^{\prime}$ of $P$ characterized by $P_{M}=-1$, while the remaining dark matter component transforms nontrivially under the quotient group $Z_{3}$, i.e., in $\underline{1}_{3}^{\prime}$ or $\underline{1}_{3}^{\prime \prime}$ of $Z_{3}$ characterized by $\left[p^{2}\right]=[w] \rightarrow w$ or $\left[p^{2}\right]=\left[w^{2}\right] \rightarrow w^{2}$, respectively.

First, to ensure the residual symmetry $Z_{6}$, i.e., $p^{6}=\exp [i 6 \pi(B-L)]=1$, every dark field should possess 
TABLE IV. $B-L$ charge of the dark field kinds that depends on which statistics they obey.

\begin{tabular}{lcc}
\hline \hline Field & Scalar & Fermion \\
\hline$[B-L]\left(F_{1}\right)$ & $1+2 n_{1}$ & $2 n_{1}$ \\
{$[B-L]\left(F_{2}\right)$} & $\pm \frac{2}{3}+2 n_{2}$ & $\pm \frac{1}{3}+2 n_{2}$ \\
{$[B-L](\Phi)$} & $\pm \frac{1}{3}+2 n_{3}$ & $\pm \frac{2}{3}+2 n_{3}$ \\
\hline \hline
\end{tabular}

a $B-L$ charge, such that $3(B-L)$ is integer. Thus, $\left[p^{2}\right]$ is nontrivial if and only if

$$
B-L= \pm \frac{1}{3}+n_{1}= \pm \frac{1}{3}, \pm \frac{2}{3}, \pm \frac{4}{3}, \pm \frac{5}{3}, \cdots
$$

for a generic field. Moreover, $P_{M}$ is odd if and only if

$$
B-L=\frac{1+2 n_{2}}{3}= \pm \frac{1}{3}, \pm 1, \pm \frac{5}{3}, \pm \frac{7}{3}, \cdots
$$

for bosonic field, and

$$
B-L=\frac{2 n_{3}}{3}=0, \pm \frac{2}{3}, \pm \frac{4}{3}, \pm 2, \cdots
$$

for fermionic field. The charge parameters $n_{1,2,3}$ are arbitrarily integer. Thus, $B-L$ is quantized with basic periods 1 and $2 / 3$ resulting from cyclic property of the residual symmetries $Z_{3}$ and $P$, respectively. Additionally, the opposite signs indicate that a field and its conjugation belong to the same kind of dark matter, i.e., nontrivial under $Z_{3}, P$, or both.

To find the minimal realistic setup of two-component dark matter, we demand that the dark sector contains spin-0 bosonic and/or spin-1/2 fermionic fields, transforming as standard model singlets. Additionally, the three kinds of dark fields, $P, Z_{3}$, and both, each have a field, called $F_{1}, \quad F_{2}$, and $\Phi$, respectively, where $F_{1} \sim(-1,1)$, $F_{2} \sim\left(1, w / w^{2}\right)$, and $\Phi \sim\left(-1, w / w^{2}\right)$ given in terms of $\left(P_{M},\left[p^{2}\right]\right)$ nontrivially transform under $P, Z_{3}$, and both, respectively. Hence their $B-L$ charge is determined in Table IV dependent on which type, either a scalar or a fermion, they are. ${ }^{2}$ Obviously, such a dark field can possess a minimal $B-L$ charge, while its real $B-L$ charge is obtained by the cyclic property. Therefore, $F_{1}$ has either $B-L=0$ as a fermion or \pm 1 as a scalar, $F_{2}$ has either $B-L= \pm 1 / 3$ as a fermion or $\pm 2 / 3$ as a scalar, and $\Phi$ has either $B-L= \pm 1 / 3$ as a scalar or $\pm 2 / 3$ as a fermion. Among these solutions based upon $P \otimes Z_{3}$, let us assume the simplest (i.e., most minimal $B-L$ ) dark matter candidates, as summarized in Table V. Note that the

\footnotetext{
${ }^{2}$ There possibly exist renormalizable couplings of $\chi$ to dark fields, such as $\chi F_{1}^{2}, \chi F_{2}^{3}, \chi F_{1} F_{2} \Phi$, and $\chi F_{2} \Phi^{2}$, with appropriate $B-L$ charges, besides the usual ones $\left(\chi^{*} \chi\right)\left(X^{*} X\right)$ for $X=F_{1}, F_{2}, \Phi$, given that all the dark fields are scalar.
}

TABLE V. Simplest dark matter candidates implied by the residual symmetry $P \otimes Z_{3}$, where the $P$ and $Z_{3}$ representations are determined by the matter parity $P_{M}$ and the quotient generator $\left[p^{2}\right]$, respectively.

\begin{tabular}{lcccc}
\hline \hline Field & $P_{M}$ & $P$ & {$\left[p^{2}\right]$} & $Z_{3}$ \\
\hline$F_{1} \sim(1,1,0,0)$ & -1 & $\underline{1}_{P}^{\prime}$ & 1 & $\underline{1}_{3}^{\prime}$ \\
$F_{2} \sim(1,1,0,1 / 3)$ & 1 & $\underline{1}_{P}$ & $w$ & $\underline{1}_{3}^{\prime}$ \\
$\Phi \sim(1,1,0,-1 / 3)$ & -1 & $\underline{1}_{P}^{\prime}$ & $w^{2}$ & $\underline{1}_{3}^{\prime \prime}$ \\
\hline \hline
\end{tabular}

$B-L$ charge of each dark field can deviate from the supplied value by an arbitrary even number-the common multiple of the basic 1 and $2 / 3$ periods - that necessarily does not change the $P, Z_{3}$ representations of the field. ${ }^{3}$ Here, $F$ and $\Phi$ mean fermion and scalar dark fields, respectively. Further, we assume the net mass of $F_{1}$ and $F_{2}$ is smaller than that of $\Phi$. In this setup, there is no coupling of a $\chi$ to two dark fields, because of the Lorentz and $U(1)_{B-L}$ invariance.

The dark matter component stabilized by $P$ (i.e., $F_{1}$ ) can have an arbitrary mass. This is also valid for the dark matter component stabilized by $Z_{3}$ (i.e., $F_{2}$ ), despite the fact that this $F_{2}$ component may be heavier than the light quarks $u$, $d$. Notice that, since all $F_{2}, u$, and $d$ transform nontrivially under $Z_{3}$, this symmetry by itself does not prevent $F_{2}$ from decay to quarks. However, when $Z_{3}$ is combined with $S U(3)_{C}$, the $F_{2}$ stability is ensured. Prove: Since the $Z_{3}$ dark matter component is color neutral, it cannot decay to any colored final state, such as those that include single quarks, because of the color conservation. The $S U(3)_{C}$ conservation requires a color-neutral final state if it results from a dark matter decay, by assumption. Obviously, this color-neutral final state if constructed from quarks must include only combinations of $q^{c} q$ and/or $q q q$ (and/or their conjugation). It follows that the final state is invariant (i.e., singlet) under $Z_{3}$ too. Because of the $Z_{3}$ conservation, such a final state cannot be the product of any $Z_{3}$ dark matter decay, which leads to a contradiction. In other words, the $S U(3)_{C}$ and $Z_{3}$ symmetries jointly suppress the decay of $Z_{3}$ dark matter component (i.e., stabilized), even if this component has a mass larger than that of a quark.

With this proposal, we have the novel, simplest model for two-component dark matter based upon $F_{1}$ and $F_{2}$ selfinteracting through a heavier dark field $\Phi$, which is of course implied by the residual symmetry $P \otimes Z_{3}$, thus the canonical seesaw. [We can have other scenarios for twocomponent dark matter, if either the alternative solutions of $F_{1,2}, \Phi$ or more dark fields are introduced and that a coupling of $\chi$ to two dark fields may arise. But they are complicated and suppressed.] Notice that since $F_{1,2}$ and $\Phi$ are the standard model singlets, the $U(1)_{B-L}$ dynamics is

\footnotetext{
${ }^{3}$ The actual $B-L$ charges of the dark fields would be phenomenologically determined.
} 
crucially/sufficiently governing the dark matter observables, besides the known consequences of neutrino mass and baryon asymmetry [25].

\section{SEESAW IMPLICATION FOR THE XENON1T EXCESS}

The XENON1T experiment has recently reported an excess in electronic recoil energy ranging from 1 to $7 \mathrm{keV}$, peaked around $2.4 \mathrm{keV}$, having a local statistical significance above $3 \sigma$ [14]. Such signal of electron recoils seems to reveal the existence of a structured dark sector [26-60]. ${ }^{4}$ Indeed, the authors in [27] first showed that in order to fit the excess, the dark matter component that scatters off electrons should be fast moving $v_{2} \sim 0.03-0.25$ for the dark matter mass $m_{2} \sim 0.1 \mathrm{MeV}$ to $10 \mathrm{GeV}$, which exceeds the velocity of cold dark matter $v_{1} \sim 10^{-3}$.

This fast dark matter component $\left(F_{2}\right)$ may be generated locally as a boosted dark matter from the annihilation or semiannihilation of the heavier dark matter component $\left(F_{1}\right)$, which is nicely implicated by our model. As a matter of fact, the heavier component with the quantum numbers $F_{1} \sim(1,1,0,0)$ interacting with normal matter only via gravity would dominate the cold dark matter, set by its annihilation or coannihilation to the lighter dark matter component $F_{2}$ through the dark matter self-interaction, $\bar{F}_{1} F_{2} \Phi$ plus its conjugation. The lighter component $F_{2}$ subdominates the dark matter abundance, since it strongly couples to the $Z^{\prime}$ portal which is totally annihilated before freeze-out.

The relevant Lagrangian terms are

$$
\begin{aligned}
\mathcal{L} \supset & \bar{F}_{1}\left(i \gamma^{\mu} \partial_{\mu}-m_{1}\right) F_{1}+\bar{F}_{2}\left(i \gamma^{\mu} D_{\mu}-m_{2}\right) F_{2} \\
& +\left[\left(D^{\mu} \Phi\right)^{\dagger}\left(D_{\mu} \Phi\right)-m_{0}^{2} \Phi^{\dagger} \Phi\right]+\bar{e}\left(i \gamma^{\mu} D_{\mu}-m_{e}\right) e \\
& +\left(h \bar{F}_{1} F_{2} \Phi+\text { H.c. }\right),
\end{aligned}
$$

where $D_{\mu}=\partial_{\mu}+i g_{B-L}(B-L) Z_{\mu}^{\prime}$ and the dark matter masses obey $m_{0}>m_{1}+m_{2}$ and $m_{1}>m_{2}$. Since the $B-$ $L$ charge of $F_{1}$ is fixed as $B-L=0$, the remaining dark fields can possess more general $B-L$ charges,

$F_{2} \sim(1,1,0,1 / 3+2 n), \quad \Phi \sim(1,1,0,-1 / 3-2 n)$,

for $n=0, \pm 1, \pm 2, \ldots$, as mentioned.

The relic density of $F_{1}$ is governed by Feynman diagrams in Fig. 1. The coannihilation process is only enhanced when the masses of $F_{1}$ and $\Phi$ are highly degenerate. However, this work signifies $m_{0}>m_{1}+m_{2} \sim$ $2 m_{1}$ such that the coannihilation contribution is negligible, where notice that $m_{2} \sim m_{1}$ is used, since $F_{2}$ is mildly boosted responsible for the XENON1T excess. Hence, the

\footnotetext{
${ }^{4}$ For other interpretations, see [61-66], for instance.
}
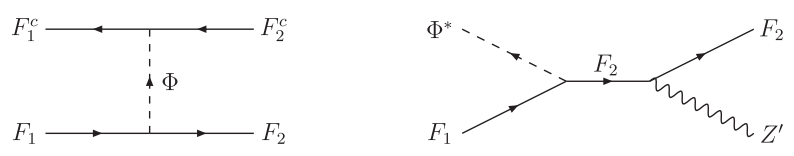

FIG. 1. Annihilation (left) and coannihilation (right) processes of $F_{1}$ that set the cold dark matter density.

dark matter abundance is given by the $F_{1}$ annihilation in the left diagram.

Applying the Feynman rules, we obtain the thermal average cross section times relative velocity as

$$
\left\langle\sigma v_{\text {rel }}\right\rangle \simeq \frac{|h|^{4}\left(m_{1}+m_{2}\right)^{2}}{8 \pi m_{0}^{4}}\left(1-\frac{m_{2}^{2}}{m_{1}^{2}}\right)^{1 / 2},
$$

which relates to the $F_{1}$ abundance, $\Omega h^{2} \simeq 0.1 \mathrm{pb} /\left\langle\sigma v_{\text {rel }}\right\rangle$, where $h$ is the reduced Hubble parameter without confusion. Using the experimental data $\Omega h^{2} \simeq 0.12$ [1] and the Lorentz boost by the left diagram $\gamma_{2}=m_{1} / m_{2}=\left(1-v_{2}^{2}\right)^{-1 / 2} \approx 1$, i.e. $m_{1} \approx m_{2}$, we get the constraint of the dark matter selfcoupling to be

$$
|h| \simeq 7 \times 10^{-4}\left(\frac{m_{0}}{2 m_{1}}\right)\left(\frac{m_{1}}{1 \mathrm{MeV}}\right)^{1 / 2}\left(\frac{\delta m^{2}}{m_{1}^{2}}\right)^{-1 / 8},
$$

which translates to $|h| \gtrsim 10^{-3}$, because of $m_{0} \gtrsim 2 m_{1}$, $m_{1} \gtrsim 1 \mathrm{MeV}$, and $\delta m^{2} / m_{1}^{2}=\left(m_{1}^{2}-m_{2}^{2}\right) / m_{1}^{2} \simeq v_{2}^{2} \sim 1 \%$ as shown below. Since the $F_{1}$ dark matter is thermally generated, its mass has been assumed to be larger than the $\mathrm{BBN}$ and $\mathrm{CMB}$ bounds $\sim 1 \mathrm{MeV}$ which are hereafter taken as the electron mass, i.e., $m_{1}>m_{e}$ [67].

Of course, at present, $F_{2}$ is locally generated by the left diagram in Fig. 1 which subsequently scatters off electrons in the XENON1T experiment through the diagram in Fig. 2. The recoil energy, deposited by the $F_{2}$-electron scattering, required to fit the excess is few $\mathrm{keV}$, i.e., $E_{R} \sim 2.4 \mathrm{keV}$. In this model, since the $F_{2}$ mass satisfies $m_{2} \approx m_{1}>m_{e}$, the electronic recoil energy is estimated as $E_{R}=E_{e^{\prime}}-$ $E_{e}=2 \mu v_{\text {rel }} v_{\mathrm{cm}}<2 m_{e} v_{2}^{2}$, where the incoming velocities of $F_{2}$ and $e$, called $v_{2}$ and $v_{e}$ respectively, are assumed to be parallel, $\mu=m_{2} m_{e} /\left(m_{2}+m_{e}\right)$ is the reduced mass, $v_{\text {rel }}=v_{2}-v_{e}$ is the relative velocity, and $v_{\mathrm{cm}}=\left(m_{2} v_{2}+\right.$ $\left.m_{e} v_{e}\right) /\left(m_{2}+m_{e}\right)$ is the center-of-mass velocity. Thus, the recoil energy is comparable to the observed value if $F_{2}$ is boosted with a velocity $v_{2} \sim 0.1$, in agreement to the best fit in [27]. This yields a transferred momentum $q=-E_{R} / v_{\mathrm{cm}}$, such that $|q| \sim 40 \mathrm{keV}$. In the limit of mediator mass $m_{Z^{\prime}}$ to

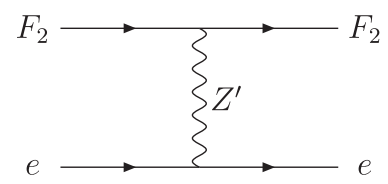

FIG. 2. Scattering process of the boosted dark matter $F_{2}$ with electrons in the XENON1T experiment. 
be much larger than the momentum transfer, $m_{Z^{\prime}} \gg|q|$, the $F_{2}$-electron scattering cross-section can be written as [68]

$$
\sigma_{e}=\frac{g_{B-L}^{4}(1 / 3+2 n)^{2} m_{e}^{2}}{\pi m_{Z^{\prime}}^{4}} .
$$

This leads to the number of the signal events as related to the scattering cross section by $[35,69]$

$$
\frac{N_{\mathrm{sig}}}{100}=\frac{16 \sigma_{e}}{3 \mathrm{pb}}\left(\frac{1 \mathrm{MeV}}{m_{1}}\right)^{2} .
$$

The mass of $Z^{\prime}$ boson is $m_{Z^{\prime}}=2 g_{B-L} \Lambda$, where the contribution of the kinetic mixing effect between the $U(1)_{Y}$ and $U(1)_{B-L}$ gauge bosons is radically small and neglected [1]. Therefore, the scattering cross section becomes $\sigma_{e}=(1 / 3+2 n)^{2} m_{e}^{2} /\left(16 \pi \Lambda^{4}\right)$, which does not explicitly depend on the $Z^{\prime}$ mass, $m_{Z^{\prime}}$, and the $Z^{\prime}$ coupling, $g_{B-L}$, but on the $B-L$ breaking scale $\Lambda=m_{Z^{\prime}} / 2 g_{B-L}$, governed by the ratio of the $Z^{\prime}$ mass to the $Z^{\prime}$ coupling. Additionally, the new observation is that the cross section is significantly enhanced by the $B-L$ charge of $F_{2}$ dark matter as multiplied by $n^{2}$ as this charge is large, but it is suppressed by the new physics scale to be $1 / \Lambda^{4}$. Correspondingly, the event number is proportional to $N_{\text {sig }} / 100 \sim\left(10^{-5} n\right)^{2}\left(m_{e} / m_{1}\right)^{2}(\mathrm{TeV} / \Lambda)^{4}$, as enhanced by $n^{2}$, but suppressed by $1 / m_{1}^{2} \times 1 / \Lambda^{4}$.

The viable regime of the dark matter charge and the new physics scale is determined by making a contour of $N_{\text {sig }} / 100=1$ from (32) as implied by the XENON1T experiment to be a function of $(n, \Lambda)$ as in Fig. 3 upper panel, taking the lower mass limit for thermal dark matter $m_{1}=m_{e} \simeq 0.51 \mathrm{MeV}$, where the collider bound $\Lambda=3 \mathrm{TeV}$ is shown (cf. Appendix A), as well as noting that the thermal dark matter excluded region corresponds to $m_{1}<m_{e}$. On the other hand, the enhancement of the event number in terms of the dark matter charge is illustrated in Fig. 3 lower panel, taking into account some allowed values of the dark matter mass and the new physics scale from the above regime:

$$
\left(m_{1}, \Lambda\right)=(0.51,3),(0.51,5),(5,3),(5,5),
$$

in $(\mathrm{MeV}, \mathrm{TeV})$, respectively.

Remarks are in order

(1) From the figure, the dark matter charge has a lower limit to be large, required in order to explain the number of the signal events, $N_{\text {sig }} / 100=1$, i.e.,

$$
|1+6 n| \simeq 915.15 \frac{m_{1} \Lambda^{2}}{\mathrm{GeV}^{3}},
$$

since both $\Lambda$ and $m_{1}$ have a lower bound, $\Lambda=3 \mathrm{TeV}$ and $m_{1}=m_{e}$, implying
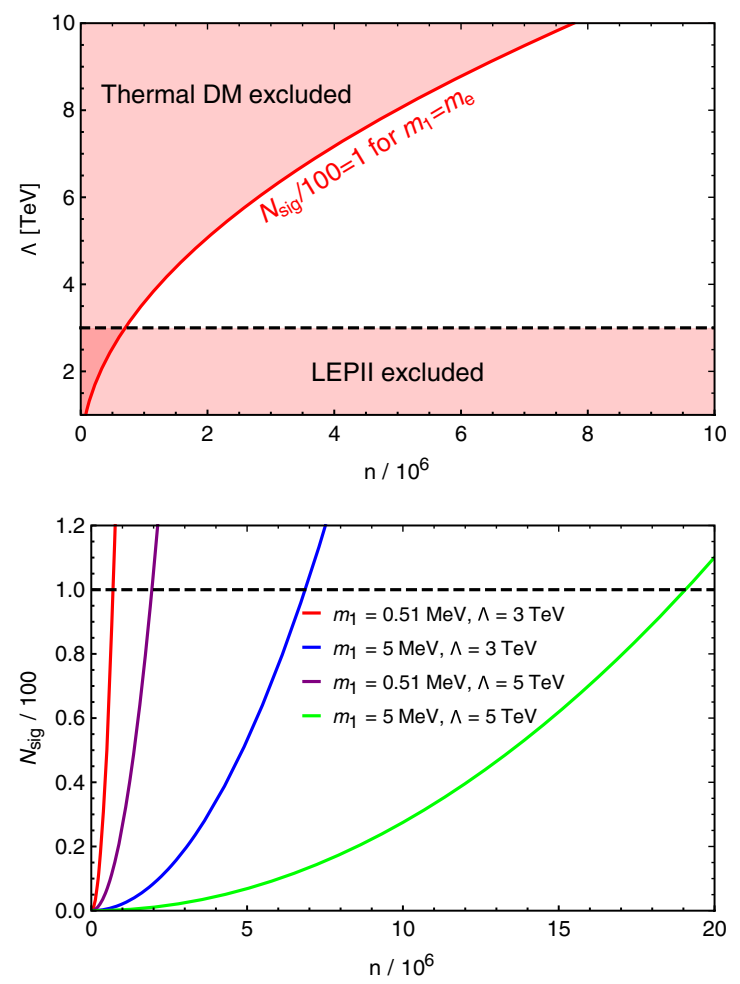

FIG. 3. Upper panel: allowed (white) region of $(n, \Lambda)$ determined by (i) contour of $N_{\text {sig }} / 100=1$ as a function of $(n, \Lambda)$ according to the WIMP mass limit $m_{1}=m_{e}$ and (ii) inclusion of the collider constraint on $\Lambda$. Lower panel: number of the signal events, $N_{\text {sig }} / 100$, significantly enhanced by the fast dark matter charge for the reference values of the thermal dark matter mass and the new physics scale.

$$
|n| \simeq 0.7 \times 10^{6}
$$

This coincides with the $n$ value in Fig. 3 at which the red line intersects either $\Lambda=3 \mathrm{TeV}$ in the upper panel or $N_{\text {sig }} / 100=1$ in the lower panel.

(2) Also from the figure, the model can generally maintain the excess in XENON1T with a stronger $B-L$ breaking scale and/or larger dark matter mass, but this requires the minimum value of $n$ to be correspondingly enhanced, which is indeed bigger than that in (35) by factors $\left(m_{1} / m_{e}\right)(\Lambda / 3 \mathrm{TeV})^{2}$. For instance, we obtain

$$
|n| \simeq 1.94 \times 10^{6}, 6.86 \times 10^{6}, 1.9 \times 10^{7},
$$

according to the last three pairs of $\left(m_{1}, \Lambda\right)$ in (33), which can be seen in Fig. 3 lower panel when the relevant curves intersect the dashed line.

The nature of a large $U(1)$ charge and the behavior of its coupling are explained in Appendix B. From the remark 1 and 2, such a minimum value of $n$ would translate to an upper bound for $g_{B-L}$ and $m_{Z^{\prime}}$, as shown below. 
(3) Obviously, the model with $n=0$ fails to account for the excess, unless the kinetic mixing and $g_{B-L}$ fine-tuning to unnaturally small values is imposed, which is not interpreted in this work.

Applying the criteria in Appendix B to $U(1)_{B-L}$, we approximate $b_{B-L} \simeq-(5 / 3)(1 / 3+2 n)^{2}$, thus

$$
\mu \partial g_{B-L}^{\prime} / \partial \mu \simeq\left(5 / 48 \pi^{2}\right) g_{B-L}^{\prime 3},
$$

which yields that $g_{B-L}^{\prime} \equiv|1 / 3+2 n| g_{B-L}$ slides similarly to the usual one. The solution is

$$
\alpha^{\prime-1}=\alpha_{G}^{\prime-1}-(5 / 6 \pi) \ln \left(\mu / \mu_{G}\right),
$$

where $\alpha^{\prime} \equiv g_{B-L}^{\prime 2} / 4 \pi$ and a subscript ${ }_{G}$ referring to that of the GUT (the Planck regime may be chosen, but not necessary). Further, one demands a perturbative limit for the $U(1)_{B-L}$ gauge interaction at the GUT scale, i.e., $\alpha_{G}^{\prime} \simeq$ 1 at $\mu_{G} \sim 10^{16} \mathrm{GeV}$, which also necessarily prevents a proton decay since it occurs only via a GUT breakdown. This leads to $\alpha^{\prime} \simeq 0.08$ at the scale of interest, $\mu \sim 1 \mathrm{MeV}{ }^{5}$ Thus, we get

$$
g_{B-L}^{\prime}=|1 / 3+2 n| g_{B-L} \simeq 1 .
$$

According to the values of $n$ in (35) and (36), the relation (39) implies upper bounds for

$$
\begin{aligned}
g_{B-L} \simeq \frac{0.5}{n} \simeq 7.1 \times 10^{-7}, 2.6 \times 10^{-7}, \\
7.2 \times 10^{-8}, 2.6 \times 10^{-8}
\end{aligned}
$$

respectively. With the aid of (34) and (39), the $Z^{\prime}$ mass is rewritten as

$$
m_{Z^{\prime}} \simeq 3.62 \times 10^{3}\left(g_{B-L} / m_{1}\right)^{1 / 2} \mathrm{MeV}^{3 / 2} .
$$

Corresponding to the above values of $\left(g_{B-L}, m_{1}\right)$, the $Z^{\prime}$ mass is bounded, respectively, by

$$
m_{Z^{\prime}} \simeq 4.3,2.6,0.43,0.26 \mathrm{MeV} .
$$

Notice that if $\left(m_{1}, \Lambda\right)$ are higher than those in (33), the $Z^{\prime}$ coupling and mass get more restricted.

Since the $Z^{\prime}$ mass is radically low, the constraints from low energy experiments apply. Because of the conditions for $m_{Z^{\prime}}$ in (C2) as well as $m_{2}<m_{0}$, the $Z^{\prime}$ boson cannot decay invisibly to any dark field, $\operatorname{Br}\left(Z^{\prime} \rightarrow F_{2}^{c} F_{2}\right)=$ $\operatorname{Br}\left(Z^{\prime} \rightarrow \Phi^{*} \Phi\right)=0$, and obviously $\operatorname{Br}\left(Z^{\prime} \rightarrow F_{1}^{c} F_{1}\right)=0$. Hence, $Z^{\prime}$ may only decay to the neutrinos $Z^{\prime} \rightarrow \nu_{L}^{c} \nu_{L}$ and

\footnotetext{
${ }^{5}$ Generally, the physical processes relevant to the gauge interactions of $F_{2}$ and $\Phi$ are determined by $\alpha^{\prime}$, thus are perturbative, since $\alpha^{\prime}$ decreases below 1 when the energy scale decreases below the GUT scale.
}

charged leptons $Z^{\prime} \rightarrow e^{+} e^{-}$if $m_{Z^{\prime}}>2 m_{e}$, being the same with the usual $U(1)_{B-L}$ model, studied in [70-76]. That said, the $U(1)_{B-L}$ gauge coupling is strongly constrained by the beam dump experiments to be roundly $g_{B-L} \sim 10^{-8}$ for $m_{Z^{\prime}}=1-10 \mathrm{MeV}$ (cf. Figs. 5 and 13 of [73] and [74], respectively). Such bound excludes the values of $g_{B-L}$ in (40) that correspond to $m_{Z^{\prime}}>2 m_{e} \simeq 1 \mathrm{MeV}$ given in (42). However, the bound does not apply to $g_{B-L}$ that reduces $m_{Z^{\prime}}$ below $1 \mathrm{MeV}$, since the detectors in such experiments are only sensitive to the signal of $Z^{\prime} \rightarrow e^{+} e^{-}$decay. Precisely, with the aid of (41), the surviving condition $m_{Z^{\prime}}<1 \mathrm{MeV}$ leads to

$$
g_{B-L}<3.9 \times 10^{-8}\left(m_{1} / m_{e}\right) .
$$

This regime excludes the processes (i) in Appendix $C$ that set the $F_{2}$ density at $Z^{\prime}$ resonance $m_{Z^{\prime}}=2 m_{2}>1 \mathrm{MeV}$, despite $\Omega_{F_{2}} h^{2} \sim\left(4 m_{2}^{2}-m_{Z^{\prime}}^{2}\right)^{2} /\left(m_{2}^{2} m_{Z^{\prime}}^{2}\right)$ tending to zero as desirable, where it yields $g_{B-L}>4 \times 10^{-8}$ in tension with the bound $g_{B-L} \sim 10^{-8}$.

Below $m_{Z^{\prime}}=1 \mathrm{MeV}$, the model is constrained by several experiments: The neutrino-electron scattering exchanged by $Z^{\prime}$ fully studied in [71] using the measurements from Borexino [77], Texono [78], and Charm-II $\left(\bar{\nu}_{\mu}\right)$ $[79,80]$; The energy loss, carried by $Z^{\prime}$, of the horizontal branch (HB) stars [81] and the supernova 1987A (SN1987A) [82,83]. Including the beam dump experiments given by the darkcast package [73] using the measurements from E137 [84], $\nu$-CAL I [85,86], Orsay [87], and E141 [88], all the relevant experimental constraints and the model prediction from (41) are plotted in Fig. 4. Hence, $m_{1}=$ $165 \mathrm{MeV}$ is the upper limit of dark matter mass determined by the HB and Borexino bounds, while it is confidently that $m_{1} \simeq m_{e} \simeq 0.51 \mathrm{MeV}$ is the lower limit of dark matter mass at which the theory approximately coincides with the E137 and SN1987A bounds, that is

$$
0.51 \mathrm{MeV}<m_{1}<165 \mathrm{MeV} .
$$

Further, our model predicts

$$
0.34 \mathrm{MeV}<m_{Z^{\prime}}<1 \mathrm{MeV}
$$

limited by the HB and the beam dump insensitivity, and

$$
3.8 \times 10^{-8}<g_{B-L}<2.9 \times 10^{-6},
$$

bounded by the SN1987A and the Borexino, respectively. Notice that the region close to the top of Fig. 4, that is bounded by the red line $m_{1}=0.51 \mathrm{MeV}$ and allowed by the Texono and Orsay experiments, is excluded by the XENON1T, since $m_{Z^{\prime}} \leq 4.3 \mathrm{MeV}$. 


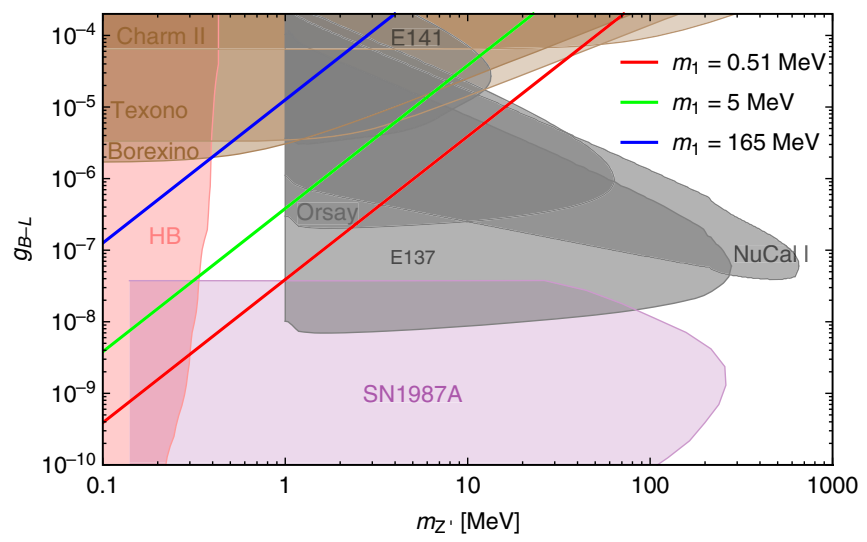

FIG. 4. Theoretical prediction (41) for dark matter mass $m_{1}=0.51,5$, and $165 \mathrm{MeV}$, where the relevant strongest experimental constraints (the color regions denote excluded parameter space according to each experiment) have been adapted from [73] for the beam dump (E137, $\nu$-CAL I, Orsay, and E141), [71] for the neutrino-electron scattering (Borexino, Texono, and Charm II with $\bar{\nu}_{\mu}$ ), [72] for the HB stars (extracted from [81]), and [70] for the SN1987A (translated from [82]), where $m_{1}=165 \mathrm{MeV}$ was chosen so that the theoretical prediction intersects the $\mathrm{HB}$ and Borexino bounds.

\section{CONCLUSION}

We have discovered a seminal result of the seesaw mechanism with gauged $B-L$ symmetry, alternative to the known leptogenesis, that it manifestly solves the longstanding issue of structured dark matter. The seesaw scale presents a nontrivial physical vacuum preserving two residual gauge symmetries, relating to the usual matter parity $P_{M}=(-1)^{3(B-L)+2 s}$ and a new $Z_{3}$ quotient $\left[p^{2}\right]=$ $\left[w^{3(B-L)}\right]$. This yields a two-component dark matter scenario naturally addressing the recent XENON1T excess. The cold dark matter $F_{1}$ has $B-L=0$, while the boosted dark matter $F_{2}$ has $B-L$ deviating from $1 / 3$ by seven order (roundly), and their masses $m_{1,2}$ obey $\operatorname{Max}\left(m_{e}, m_{Z^{\prime}}\right)<m_{1} \approx m_{2}<$ $165 \mathrm{MeV}$. The $Z^{\prime}$ mass and coupling predicted are $0.34 \mathrm{MeV}<m_{Z^{\prime}}<1 \mathrm{MeV}$ and $3.8 \times 10^{-8}<g_{B-L}<$ $2.9 \times 10^{-6}$, respectively. If the XENON1T experiment is relaxed, a scheme of two-component dark matter beyond the weak scale is warranted, presented in standard model gauge extensions containing a $B-L$ charge, such as the left-right symmetry and $S O(10)$.

\section{ACKNOWLEDGMENTS}

This research is funded by Vietnam National Foundation for Science and Technology Development (NAFOSTED) under Grant No. 103.01-2019.353.

\section{APPENDIX A: HIGH ENERGY COLLIDER CONSTRAINT}

Contribution of $Z^{\prime}$ to the process $e^{+} e^{-} \rightarrow f \bar{f}$, where $f$ is an ordinary fermion, proceeds through the $Z^{\prime}$ exchange either by $s$-channel diagram for $f \neq e$ or by $s, t, u$-channel diagrams for $f=e$.

If $m_{Z^{\prime}}<\sqrt{s}=209 \mathrm{GeV}$ as in this model, the $Z^{\prime}$ boson is resonantly produced at the LEPII and the agreement between the LEPII and the standard model indicates that the $Z^{\prime}$-fermion couplings are $g_{B-L} \lesssim 10^{-2}$, given that $Z^{\prime}$ does not decay to the dark fields $F_{2}, \Phi$ [89]. However, when $m_{Z^{\prime}}>2 m_{F_{2}, \Phi}$, the decay to the dark fields dominates the $Z^{\prime}$ width and then the cross section $\sigma\left(e^{+} e^{-} \rightarrow f \bar{f}\right)=$ $\sigma\left(e^{+} e^{-} \rightarrow Z^{\prime}\right) \operatorname{Br}\left(Z^{\prime} \rightarrow f \bar{f}\right)$ is strongly suppressed, since $\operatorname{Br}\left(Z^{\prime} \rightarrow f \bar{f}\right) \sim 1 / n^{2} \ll 1$. This case requiring $g_{B-L} / n \lesssim$ $10^{-2}$ is obviously satisfied for the $n$ value of interest and $g_{B-L}$ in perturbative regime.

By contrast, if $m_{Z^{\prime}}>\sqrt{s}=209 \mathrm{GeV}$, the process $e^{+} e^{-} \rightarrow f \bar{f}$ receives an off-shell contribution of $Z^{\prime}$, best described by the effective interaction,

$$
\mathcal{L}_{\text {eff }} \supset \frac{g_{B-L}^{2}(B-L)_{f}}{m_{Z^{\prime}}^{2}-s}\left(\bar{e} \gamma^{\nu} e\right)\left(\bar{f} \gamma_{\nu} f\right) .
$$

Considering $f=\mu, \tau$, the LEPII has limited the $Z^{\prime}$ mass over coupling to be $m_{Z^{\prime}} / g_{B-L} \geq 6 \mathrm{TeV}$, which translates to the breaking scale $\Lambda \geq 3 \mathrm{TeV}[90,91]$.

Additionally, the LHC has searched for dilepton signals $p p \rightarrow \bar{l}$ as mediated by $Z^{\prime}$, yielding a mass limit roundly $m_{Z^{\prime}}=4 \mathrm{TeV}$ for the $Z^{\prime}$ coupling identical to $Z$, which converts to a bound for $\Lambda \sim m_{Z^{\prime}} / 2 g \sim 3 \mathrm{TeV}$, similar to the LEPII [92]. Here $Z^{\prime}$ does not decay to the dark matter. Otherwise, the cross section is more suppressed, similar to the LEPII case.

As a matter of fact, $Z^{\prime}$ cannot decay to the dark fields in this model [cf. (C2)]. The production of mono- $X$ or two- $X$ 's final state (signature) recoils against large missing momentum (or energy) carried by dark matter is insensitive to the LEPII and LHC detectors, because of the suppression $\operatorname{Br}\left(Z^{\prime} \rightarrow \mathrm{DM} \mathrm{DM}\right)=0$.

In short, these experiments give a reference of the new physics scale, say $\Lambda=3 \mathrm{TeV}$, used in the body text.

\section{APPENDIX B: NATURE OF $U(1)$ CHARGE AND RUNNING COUPLING}

In contrast to the non-Abelian charges (e.g., the color and the weak isospin) that are constrained by the nonAbelian nature of a Lie algebra, $\left[T_{j}, T_{k}\right]=i f_{j k l} T_{l}$ with $\operatorname{Tr}\left(T_{j} T_{k}\right) \sim \delta_{j k}$, the Abelian charges including electric charge are completely arbitrary, often chosen to describe observed charge values, while do not explain them.

Indeed, the Hamiltonian of a $U(1)_{X}$ gauge theory preserves a scaling symmetry, $g_{X} \rightarrow g_{X}^{\prime}=g_{X} / \lambda$ and $X \rightarrow X^{\prime}=\lambda X$, where $g_{X}^{\prime}$ and $X^{\prime}$ are the respective coupling and charge after the transformation. We can work in a basis that $g_{X}$ is small, while $X$ is large, which leaves the physics unchanged. 
For instance, the running of $g_{X}$ with renormalization scale $\mu$ satisfies the RG equation,

$$
\mu \partial g_{X} / \partial \mu=\beta\left(g_{X}\right)=-\left(g_{X}^{3} / 16 \pi^{2}\right) b_{X}
$$

where the beta function at 1-loop level is given by

$$
b_{X}=-\frac{2}{3} \sum_{L} X_{L}^{2}-\frac{2}{3} \sum_{R} X_{R}^{2}-\frac{1}{3} \sum_{S} X_{S}^{2}
$$

as summed over the left/right chiral fermion and scalar fields, respectively. Equation (B1) conserves the above scaling symmetry as a result, implying that a theory with large $X$ behaves similarly to the usual ones.

A character of the $U(1)$ theory is that the function $b_{X}<0$ for every $X$-charge. Therefore, its gauge coupling $g_{X}$ decreases when the energy scale $\mu$ decreases. Given that the theory is definite at a GUT or Planck scale, i.e., $g_{X} X \sim 1$, it must properly work to be perturbative and predictive below such large scale.

\section{APPENDIX C: CONDITION OF VANISHED $F_{2}$ DENSITY}

Last, but not least, it is verified that the $F_{2}$ relic density should be negligible. In the early universe, the dark field $F_{2}$ might completely annihilate to (i) the standard model particles such as $F_{2}^{c} F_{2} \rightarrow l^{+} l^{-}, \nu_{L}^{c} \nu_{L}$ via the (s-channel) $Z^{\prime}$ portal or to (ii) the new gauge boson $F_{2}^{c} F_{2} \rightarrow Z^{\prime} Z^{\prime}$ via $t$ - and $u$-channel diagrams mediated by just $F_{2}$, if kinetically allowed.

If $m_{2}<m_{Z^{\prime}}$, the annihilation proceeds through the first processes (i), yielding the cross sections

$$
\left\langle\sigma v_{\mathrm{rel}}\right\rangle_{F_{2}^{c} F_{2} \rightarrow e^{+} e^{-}, \nu_{L}^{c} \nu_{L}} \sim \begin{cases}\left(\frac{m_{Z^{\prime}}}{2 \mathrm{MeV}}\right)^{4} \mathrm{pb}, & \text { if } m_{2}>\frac{1}{2} m_{Z^{\prime}} \\ \left(\frac{m_{2}}{1 \mathrm{MeV}}\right)^{4} \mathrm{pb}, & \text { if } m_{2}<\frac{1}{2} m_{Z^{\prime}}\end{cases}
$$

with the help of (34) as well as $m_{1} \approx m_{2}$, where the resonance $m_{2}=\frac{1}{2} m_{Z^{\prime}}$ need not necessarily be considered since it sets a lowest $F_{2}$ relic density. Although one vertex (namely $\bar{F}_{2} F_{2} Z^{\prime}$ ) is enhanced by the coupling strength $g_{B-L}^{\prime}=|1 / 3+2 n| g_{B-L} \simeq 1$, these contributions are only effective if $\operatorname{Min}\left\{m_{2}, \frac{1}{2} m_{Z^{\prime}}\right\}>1 \mathrm{MeV}$. In this case, since $m_{1} \approx m_{2}>1 \mathrm{MeV}$, we obtain the $Z^{\prime}$ mass bound to be $m_{Z^{\prime}}<2.2 \mathrm{MeV}$ due to (41), (39), and (34) for $\Lambda \geq 3 \mathrm{TeV}$. That said, the above possibilities give a finite contribution to dark matter, since $\left\langle\sigma v_{\text {rel }}\right\rangle_{F_{2}^{c} F_{2} \rightarrow e^{+} e^{-}, \nu_{L}^{c} \nu_{L}} \sim 1 \mathrm{pb}$, except for the resonance regime at which the $F_{2}$ density almost vanishes.

By contrast, if $m_{2}>m_{Z^{\prime}}$, the last process (ii) produces a large annihilation cross section to be

$$
\left\langle\sigma v_{\text {rel }}\right\rangle_{F_{2}^{c} F_{2} \rightarrow Z^{\prime} Z^{\prime}} \simeq \frac{|M|^{2}}{16 \pi s} \gg 1 \mathrm{pb},
$$

because the amplitude $M\left(F_{2}^{c} F_{2} \rightarrow Z^{\prime} Z^{\prime}\right) \simeq 4 g_{B-L}^{\prime 4}$ that is induced in the order of unity is strongly enhanced by both the $\bar{F}_{2} F_{2} Z^{\prime}$ vertices with the coupling strength $g_{B-L}^{\prime}=$ $|1 / 3+2 n| g_{B-L} \simeq 1$ and that $s \simeq 4 m_{2}^{2}<\mathrm{GeV}^{2}$.

Summarizing all the relevant cross sections leads to $\Omega_{F_{2}} h^{2} \simeq 0.1 \mathrm{pb} /\left\langle\sigma v_{\text {rel }}\right\rangle_{F_{2}^{c} F_{2} \rightarrow \text { all }} \ll 0.12$, provided that

$$
m_{2}>m_{Z^{\prime}} \text {, or else } m_{2}=\frac{1}{2} m_{Z^{\prime}}
$$

[1] Particle Data Group Collaboration, Review of particle physics, Phys. Rev. D 98, 030001 (2018).

[2] P. Minkowski, $\mu \rightarrow e \gamma$ at a rate of one out of $10^{9}$ muon decays? Phys. Lett. 67B, 421 (1977).

[3] M. Gell-Mann, P. Ramond, and R. Slansky, Complex spinors and unified theories, Conf. Proc. C 790927, 315 (1979).

[4] T. Yanagida, Horizontal symmetry and masses of neutrinos, Conf. Proc. C 7902131, 95 (1979).

[5] S. L. Glashow, The future of elementary particle physics, NATO Sci. Ser. B 61, 687 (1980).

[6] R. N. Mohapatra and G. Senjanovic, Neutrino Mass and Spontaneous Parity Violation, Phys. Rev. Lett. 44, 912 (1980).

[7] R. N. Mohapatra and G. Senjanovic, Neutrino masses and mixings in gauge models with spontaneous parity violation, Phys. Rev. D 23, 165 (1981).
[8] G. Lazarides, Q. Shafi, and C. Wetterich, Proton lifetime and fermion masses in an SO(10) model, Nucl. Phys. B181, 287 (1981).

[9] J. Schechter and J. W. F. Valle, Neutrino masses in $\mathrm{SU}(2) \times$ U(1) theories, Phys. Rev. D 22, 2227 (1980).

[10] J. Schechter and J. W. F. Valle, Neutrino decay and spontaneous violation of lepton number, Phys. Rev. D 25, 774 (1982).

[11] A. Davidson, $B-L$ as the fourth color within an $S U(2)_{L} \times$ $U(1)_{R} \times U(1)$ model, Phys. Rev. D 20, 776 (1979).

[12] R. E. Marshak and R. N. Mohapatra, Quark-lepton symmetry and B-L as the U(1) generator of the electroweak symmetry group, Phys. Lett. 91B, 222 (1980).

[13] R. N. Mohapatra and R. E. Marshak, Local B-L Symmetry of Electroweak Interactions, Majorana Neutrinos and Neutron Oscillations, Phys. Rev. Lett. 44, 1316 (1980). 
[14] XENON collaboration, Excess electronic recoil events in XENON1T, Phys. Rev. D 102, 072004 (2020).

[15] L. M. Krauss and F. Wilczek, Discrete Gauge Symmetry in Continuum Theories, Phys. Rev. Lett. 62, 1221 (1989).

[16] S. P. Martin, Some simple criteria for gauged R-parity, Phys. Rev. D 46, R2769 (1992).

[17] B. Batell, Dark discrete gauge symmetries, Phys. Rev. D 83, 035006 (2011).

[18] E. Ma, Derivation of Dark Matter Parity from Lepton Parity, Phys. Rev. Lett. 115, 011801 (2015).

[19] E. Ma, N. Pollard, R. Srivastava, and M. Zakeri, Gauge $B-L$ model with residual $Z_{3}$ symmetry, Phys. Lett. B 750, 135 (2015).

[20] M. Hirsch, R. Srivastava, and J. W. F. Valle, Can one ever prove that neutrinos are Dirac particles? Phys. Lett. B 781, 302 (2018).

[21] C. Bonilla, S. Centelles-Chuliá, R. Cepedello, E. Peinado, and R. Srivastava, Dark matter stability and Dirac neutrinos using only Standard Model symmetries, Phys. Rev. D 101, 033011 (2020).

[22] J. Heeck and W. Rodejohann, Neutrinoless quadruple beta decay, Europhys. Lett. 103, 32001 (2013).

[23] C. Cai, Z. Kang, H.-H. Zhang, and Y.-P. Zeng, Minimal dark matter in $S U(2)_{L} x U(1)_{Y} x U(1)_{B-L}$, Phys. Lett. B 784, 385 (2018).

[24] D. Nanda and D. Borah, Connecting light Dirac neutrinos to a multi-component dark matter scenario in gauged $B-L$ model, Eur. Phys. J. C 80, 557 (2020).

[25] M. Fukugita and T. Yanagida, Baryogenesis without grand unification, Phys. Lett. B 174, 45 (1986).

[26] F. Takahashi, M. Yamada, and W. Yin, XENON1T Excess from Anomaly-Free Axionlike Dark Matter and Its Implications for Stellar Cooling Anomaly, Phys. Rev. Lett. 125, 161801 (2020).

[27] K. Kannike, M. Raidal, H. Veermäe, A. Strumia, and D. Teresi, Dark matter and the XENON1T electron recoil excess, Phys. Rev. D 102, 095002 (2020).

[28] G. Choi, M. Suzuki, and T. T. Yanagida, XENON1T anomaly and its implication for decaying warm dark matter, Phys. Lett. B 811, 135976 (2020).

[29] J. Buch, M. A. Buen-Abad, J. Fan, and J. S. C. Leung, Galactic origin of relativistic bosons and XENON1T excess, J. Cosmol. Astropart. Phys. 10 (2020) 051.

[30] Y. Chen, J. Shu, X. Xue, G. Yuan, and Q. Yuan, Sun heated MeV-scale dark matter and the XENON1T electron recoil excess, arXiv:2006.12447.

[31] N. F. Bell, J. B. Dent, B. Dutta, S. Ghosh, J. Kumar, and J. L. Newstead, Explaining the XENON1T Excess with Luminous Dark Matter, Phys. Rev. Lett. 125, 161803 (2020).

[32] M. Du, J. Liang, Z. Liu, V. Q. Tran, and Y. Xue, On-shell mediator dark matter models and the Xenon1T excess, Chin. Phys. C 45, 013114 (2021).

[33] L. Su, W. Wang, L. Wu, J. M. Yang, and B. Zhu, Atmospheric dark matter and Xenon1T excess, Phys. Rev. D 102, 115028 (2020).

[34] K. Harigaya, Y. Nakai, and M. Suzuki, Inelastic dark matter electron scattering and the XENON1T excess, Phys. Lett. B 809, 135729 (2020).
[35] B. Fornal, P. Sandick, J. Shu, M. Su, and Y. Zhao, Boosted Dark Matter Interpretation of the XENON1T Excess, Phys. Rev. Lett. 125, 161804 (2020).

[36] G. Alonso-Álvarez, F. Ertas, J. Jaeckel, F. Kahlhoefer, and L. J. Thormaehlen, Hidden photon dark matter in the light of XENON1T and stellar cooling, J. Cosmol. Astropart. Phys. 11 (2020) 029.

[37] Y. Jho, J.-C. Park, S. C. Park, and P.-Y. Tseng, Leptonic new force and cosmic-ray boosted dark matter for the XENON1T excess, Phys. Lett. B 811, 135863 (2020).

[38] M. Baryakhtar, A. Berlin, H. Liu, and N. Weiner, Electromagnetic signals of inelastic dark matter scattering, arXiv:2006.13918.

[39] I. M. Bloch, A. Caputo, R. Essig, D. Redigolo, M. Sholapurkar, and T. Volansky, Exploring new physics with $\mathrm{O}$ $(\mathrm{keV})$ electron recoils in direct detection experiments, J. High Energy Phys. 01 (2021) 178.

[40] G. Paz, A. A. Petrov, M. Tammaro, and J. Zupan, Shining dark matter in Xenon1T, Phys. Rev. D 103, L051703 (2021).

[41] Q.-H. Cao, R. Ding, and Q.-F. Xiang, Exploring for sub$\mathrm{MeV}$ boosted dark matter from Xenon electron direct detection, Chin. Phys. C 45, 045002 (2021).

[42] H. M. Lee, Exothermic dark matter for XENON1T excess, J. High Energy Phys. 01 (2021) 019.

[43] K. Nakayama and Y. Tang, Gravitational production of hidden photon dark matter in light of the XENON1T excess, Phys. Lett. B 811, 135977 (2020).

[44] R. Primulando, J. Julio, and P. Uttayarat, Collider constraints on a dark matter interpretation of the XENON1T excess, Eur. Phys. J. C 80, 1084 (2020).

[45] G. B. Gelmini, V. Takhistov, and E. Vitagliano, Scalar direct detection: In-medium effects, Phys. Lett. B 809, 135779 (2020).

[46] J. Bramante and N. Song, Electric But Not Eclectic: Thermal Relic Dark Matter for the XENON1T Excess, Phys. Rev. Lett. 125, 161805 (2020).

[47] L. Zu, G.-W. Yuan, L. Feng, and Y.-Z. Fan, Mirror dark matter and electronic recoil events in XENON1T, Nucl. Phys. B965, 115369 (2021).

[48] S. Baek, J. Kim, and P. Ko, XENON1T excess in local $Z_{2}$ DM models with light dark sector, Phys. Lett. B 810 (2020) 135848.

[49] H. Alhazmi, D. Kim, K. Kong, G. Mohlabeng, J.-C. Park, and S. Shin, Implications of the XENON1T excess on the dark matter interpretation, arXiv:2006.16252.

[50] W. Chao, Y. Gao, and M. J. Jin, Pseudo-dirac dark matter in XENON1T, arXiv:2006.16145.

[51] L. Delle Rose, G. Hütsi, C. Marzo, and L. Marzola, Impact of loop-induced processes on the boosted dark matter interpretation of the XENON1T excess, J. Cosmol. Astropart. Phys. 02 (2021) 031.

[52] P. Ko and Y. Tang, Semi-annihilating $Z_{3}$ dark matter for XENON1T excess, Phys. Lett. B 815, 136181 (2021).

[53] H. An and D. Yang, Direct detection of freeze-in inelastic dark matter, arXiv:2006.15672.

[54] N. Okada, S. Okada, D. Raut, and Q. Shafi, Dark matter $Z^{\prime}$ and XENON1T excess from $U(1)_{X}$ extended standard model, Phys. Lett. B 810, 135785 (2020). 
[55] D. Choudhury, S. Maharana, D. Sachdeva, and V. Sahdev, Dark matter, muon anomalous magnetic moment, and the XENON1T excess, Phys. Rev. D 103, 015006 (2021).

[56] G. Arcadi, A. Bally, F. Goertz, K. Tame-Narvaez, V. Tenorth, and S. Vogl, EFT interpretation of XENON1T electron recoil excess: Neutrinos and dark matter, Phys. Rev. D 103, 023024 (2021).

[57] H.-J. He, Y.-C. Wang, and J. Zheng, EFT approach of inelastic dark matter for Xenon electron recoil detection, J. Cosmol. Astropart. Phys. 01 (2021) 042.

[58] U. K. Dey, T. N. Maity, and T. S. Ray, Prospects of migdal effect in the explanation of XENON1T electron recoil excess, Phys. Lett. B 811, 135900 (2020).

[59] J. Smirnov and J. F. Beacom, New Freezeout Mechanism for Strongly Interacting Dark Matter, Phys. Rev. Lett. 125, 131301 (2020).

[60] G. Choi, T. T. Yanagida, and N. Yokozaki, Feebly interacting $U(1)_{B-L}$ gauge boson warm dark matter and XENON1T anomaly, Phys. Lett. B 810, 135836 (2020).

[61] O. G. Miranda, D. K. Papoulias, M. Tórtola, and J. W. F. Valle, XENON1T signal from transition neutrino magnetic moments, Phys. Lett. B 808, 135685 (2020).

[62] M. Lindner, Y. Mambrini, T. B. de Melo, and F. S. Queiroz, XENON1T anomaly: A light Z' from a two Higgs doublet model, Phys. Lett. B 811, 135972 (2020).

[63] D. A. Sierra, V. De Romeri, L. Flores, and D. Papoulias, Light vector mediators facing XENON1T data, Phys. Lett. B 809, 135681 (2020).

[64] C. Boehm, D. G. Cerdeno, M. Fairbairn, P. A. Machado, and A. C. Vincent, Light new physics in XENON1T, Phys. Rev. D 102, 115013 (2020).

[65] A. N. Khan, Can nonstandard neutrino interactions explain the XENON1T spectral excess? Phys. Lett. B 809, 135782 (2020).

[66] A. Bally, S. Jana, and A. Trautner, Neutrino Self-Interactions and XENON1T Electron Recoil Excess, Phys. Rev. Lett. 125, 161802 (2020).

[67] N. Sabti, J. Alvey, M. Escudero, M. Fairbairn, and D. Blas, Refined bounds on $\mathrm{MeV}$-scale thermal dark sectors from BBN and the CMB, J. Cosmol. Astropart. Phys. 01 (2020) 004.

[68] A. Joglekar, N. Raj, P. Tanedo, and H.-B. Yu, Relativistic capture of dark matter by electrons in neutron stars, Phys. Lett. B 809, 135767 (2020).

[69] K. Agashe, Y. Cui, L. Necib, and J. Thaler, (In)direct detection of boosted dark matter, J. Cosmol. Astropart. Phys. 10 (2014) 062.

[70] R. Harnik, J. Kopp, and P. A. N. Machado, Exploring nu signals in dark matter detectors, J. Cosmol. Astropart. Phys. 07 (2012) 026.

[71] S. Bilmis, I. Turan, T. M. Aliev, M. Deniz, L. Singh, and H. T. Wong, Constraints on dark photon from neutrino-electron scattering experiments, Phys. Rev. D 92, 033009 (2015).

[72] K. Kaneta, Z. Kang, and H.-S. Lee, Right-handed neutrino dark matter under the $B-L$ gauge interaction, J. High Energy Phys. 02 (2017) 031.

[73] P. Ilten, Y. Soreq, M. Williams, and W. Xue, Serendipity in dark photon searches, J. High Energy Phys. 06 (2018) 004.

[74] M. Bauer, P. Foldenauer, and J. Jaeckel, Hunting all the hidden photons, J. High Energy Phys. 07 (2018) 094.
[75] M. Lindner, F. S. Queiroz, W. Rodejohann, and X.-J. Xu, Neutrino-electron scattering: General constraints on $Z^{\prime}$ and dark photon models, J. High Energy Phys. 05 (2018) 098.

[76] N. Okada, S. Okada, and Q. Shafi, Light $Z^{\prime}$ and dark matter from $\mathrm{U}(1)_{X}$ gauge symmetry, Phys. Lett. B 810, 135845 (2020).

[77] G. Bellini et al., Precision Measurement of the 7Be Solar Neutrino Interaction Rate in Borexino, Phys. Rev. Lett. 107, 141302 (2011).

[78] TEXONO Collaboration, Measurement of neutrino-electron scattering cross section with a $\mathrm{CsI}(\mathrm{Tl})$ scintillating crystal array at the Kuo-Sheng nuclear power reactor, Phys. Rev. D 81, 072001 (2010).

[79] CHARM-II Collaboration, Measurement of differential cross sections for muon-neutrino electron scattering, Phys. Lett. B 302, 351 (1993).

[80] CHARM-II Collaboration, Precision measurement of electroweak parameters from the scattering of muonneutrinos on electrons, Phys. Lett. B 335, 246 (1994).

[81] J. Redondo and G. Raffelt, Solar constraints on hidden photons re-visited, J. Cosmol. Astropart. Phys. 08 (2013) 034 .

[82] J. B. Dent, F. Ferrer, and L. M. Krauss, Constraints on light hidden sector gauge bosons from supernova cooling, arXiv:1201.2683.

[83] D. Kazanas, R. N. Mohapatra, S. Nussinov, V. L. Teplitz, and Y. Zhang, Supernova bounds on the dark photon using its electromagnetic decay, Nucl. Phys. B890, 17 (2014).

[84] J. D. Bjorken, S. Ecklund, W. R. Nelson, A. Abashian, C. Church, B. Lu et al., Search for neutral metastable penetrating particles produced in the SLAC beam dump, Phys. Rev. D 38, 3375 (1988).

[85] J. Blumlein et al., Limits on neutral light scalar and pseudoscalar particles in a proton beam dump experiment, Z. Phys. C 51 (1991) 341.

[86] J. Blumlein et al., Limits on the mass of light (pseudo)scalar particles from Bethe-Heitler $e+e-$ and $m u+m u-$ pair production in a proton-iron beam dump experiment, Int. J. Mod. Phys. A 07, 3835 (1992).

[87] M. Davier and H. N. Ngoc, An unambiguous search for a light Higgs boson, Phys. Lett. B 229, 150 (1989).

[88] E. M. Riordan et al., A Search for Short Lived Axions in an Electron Beam Dump Experiment, Phys. Rev. Lett. 59, 755 (1987).

[89] T. Appelquist, B. A. Dobrescu, and A. R. Hopper, Nonexotic neutral gauge bosons, Phys. Rev. D 68, 035012 (2003).

[90] ALEPH, DELPHI, L3, OPAL, LEP Electroweak Working Group Collaborations, A combination of preliminary electroweak measurements and constraints on the standard model, arXiv:hep-ex/0612034.

[91] M. Carena, A. Daleo, B. A. Dobrescu, and T. M. P. Tait, $Z^{\prime}$ gauge bosons at the Tevatron, Phys. Rev. D 70, 093009 (2004).

[92] ATLAS Collaboration, Search for new high-mass phenomena in the dilepton final state using $36 \mathrm{fb}^{-1}$ of proton-proton collision data at $\sqrt{s}=13 \mathrm{TeV}$ with the ATLAS detector, J. High Energy Phys. 10 (2017) 182. 